\title{
Erratum
}

\section{Determinants of household vulnerability to food insecurity during COVID-19 lockdown in a mid-term period in Iran - ERRATUM}

\author{
Mohammad Reza Pakravan-Charvadeh*, Moselm Savari, Haider A Khan, \\ Saeid Gholamrezai and Cornelia Flora
}

DOI: 10.1017/S1368980021000318, Published online by Cambridge University Press, 26 January 2021.

During production of the above-mentioned article, Mohammad Reza Pakravan-Charvadeh was inadvertently attributed with all four author affiliations. The only correct one is Lorestan University.

This has since been corrected. Cambridge University Press apologise for this oversight.

\section{Reference}

Pakravan-Charvadeh, M., Savari, M., Khan, H., Gholamrezai, S., \& Flora, C. (2021). Determinants of household vulnerability to food insecurity during COVID-19 lockdown in a mid-term period in Iran. Public Health Nutrition 24, 1619-1628. doi: 10.1017/ S1368980021000318 